\title{
High-resolution frequency estimation technique for recovering phase distribution in interferometers
}

\author{
Abhijit Patil and Pramod Rastogi \\ Applied Computing and Mechanics Laboratory, Ecole Polytechnique Fédérale de Lausanne, 1015-Lausanne, Switzerland \\ Rajesh Langoju \\ Biomedical Imaging Group, Ecole Polytechnique Fédérale de Lausanne, 1015-Lausanne, Switzerland
}

Received July 15, 2004

\begin{abstract}
An integral approach to phase measurement is presented. First, the use of a high-resolution technique for the pixelwise detection of phase steps is proposed. Next, the robustness of the algorithm that is developed is improved by incorporation of a denoising procedure during spectral estimation. The pixelwise knowledge of phase steps is then applied to the Vandermonde system of equations for retrieval of phase values at each pixel point. Conceptually, our proposal involves the design of an annihilating filter that has zeros at the frequencies associated with the polynomial that describes the fringe intensity. The parametric estimation of this annihilating filter yields the desired spectral information embedded in the signal, which in our case represents the phase steps. The proposed method offers the advantage of extracting the interference phase of nonsinusoidal waveforms in the presence of miscalibration error of the piezoelectric transducer. In addition, in contrast to previously reported methods, this method does not require the application of selective phase steps between data frames for nonsinusoidal waveforms. (c) 2005 Optical Society of America

OCIS codes: $120.3180,120.5050$.
\end{abstract}

Phase shifting has now become a well-established technique in optical interferometry for the detection of interference phase. The technique functions by recording $N$ frames of intensity data shifted in phase with respect to one another. The phase shifts are provided by a piezoelectric transducer (PZT). One solves the set of intensity equations at each pixel location to compute the phase. However, one of the most significant sources of error in computing this phase distribution is inaccurate calibration of the PZT. ${ }^{1}$ This problem is compounded further by aging of the PZT, environmental changes, detector nonlinearity, and multiple beam interference. ${ }^{2}$ Remedial methods have been proposed. ${ }^{1-5}$ The algorithm developed by Carré, ${ }^{3}$ although it is suitable for sinusoidal waveforms and first-order calibration errors of the PZT, needs a careful selection of phase steps for optimum measurements. Surrel ${ }^{1}$ and Larkin and Oreb $^{4}$ proposed algorithms that minimize calibration errors for sinusoidal fringes. The algorithm proposed by Hibino et al. ${ }^{5}$ offers the possibility of reducing calibration error in the presence of higher-order harmonics. Hibino et al. ${ }^{5}$ and Surrel $^{2}$ showed that $\kappa$ th-order harmonics can be minimized with phase step $2 \pi /(\kappa+2)$ between acquired data frames.

The objective of this Letter is to propose a novel approach to obtaining phase measurements in the presence of PZT miscalibration and multiple-order harmonics in the sampled waveform. This method, which permits free choice of the phase shifts from 0 to $\pi$, completely avoids imposing conditions on the phase shift that must be applied by establishing a high-resolution technique ${ }^{6}$ for estimating the phase step. The method works by drawing an analogy between the frequency that is present in the spectrum and the linear phase steps that result from use of the PZT. The method basically explores the fact that a polynomial can be associated with periodic intensity fringes recorded with a CCD camera. We have designed another polynomial, called an annihilating filter, that has zeros at frequencies associated with the polynomial that describes the intensity fringes. As a result, the discrete convolution between the filter and the intensity yields zero. A parametric estimation of this annihilating filter yields the desired spectral information that is embedded in the signal, i.e., in our case, the phase steps. Although it is well known that a fast Fourier transform is an efficient tool for the estimation of well-separated frequencies, the separation of closely spaced frequencies in the presence of noise and fewer samples can be handled with high-resolution techniques. ${ }^{7}$ The number of samples required for estimating the phase steps in our case to minimize the effect of the $\kappa$ th-order harmonic is $4 \kappa+2$. The presence of noise necessitates applying denoising techniques to permit the phase step to be estimated with reasonable accuracy. Once the phase steps are estimated at each location of the $N$ data frames, we have a linear system that is solved by use of a Vandermonde system of equations to recover the phase distribution. The proposed method has the added advantage of working with diverging as well as converging beams. We test the robustness of the method by introducing noise during the spectral estimation.

The intensity for the $N$ data frames acquired with the CCD camera can be written as

$$
\begin{aligned}
I(m)= & \beta_{0}+\sum_{k=1}^{\kappa} a_{k} \exp [i k(\varphi+m \alpha)] \\
& +\sum_{k=-1}^{-\kappa} a_{k} \exp [i k(\varphi+m \alpha)], \\
m= & 1,2, \ldots, N
\end{aligned}
$$

where $\varphi$ is the phase of the interference fringes, $\alpha$ is the phase step, $a_{k}$ is the complex Fourier coefficient of the $\kappa$ th harmonic, $i=\sqrt{-1}$, and $\beta_{0}$ is the local average 
value for intensity. Equation (1) can be rewritten as

$$
\begin{aligned}
I(m) & =\beta_{0}+\sum_{k=1}^{\kappa} \beta_{k} z_{k}{ }^{m}+\sum_{k=1}^{\kappa} \beta_{k}{ }^{*}\left(z_{k}{ }^{*}\right)^{m}, \\
m & =1,2, \ldots, N,
\end{aligned}
$$

where $\beta_{k}=a_{k} \exp (i k \varphi), \quad z_{k}=\exp (i k \alpha), \beta_{k}{ }^{*}=$ $a_{k} \exp (-i k \varphi)$, and $z_{k}{ }^{*}=\exp (-i k \alpha)$. Parameters $z_{k}$ and $\beta_{k}$ can be estimated by high-resolution analysis. This estimation is based on the observation that signal $I(m)$ can be annihilated by a specific filter:

$$
P(z)=(z-1) \prod_{k=1}^{\kappa}\left(z-z_{k}\right)\left(z-z_{k}^{*}\right)=\sum_{k=0}^{2 \kappa+1} P_{k} z^{k} .
$$

Then the discrete convolution of $P_{m} * I_{m}$ vanishes identically, i.e.,

$$
\sum_{k=0}^{2 \kappa+1} P_{k} I_{m-k}=0 \quad \forall m \in\{2 \kappa+2,2 \kappa+3, \ldots, N\} .
$$

Equation (4) can be written in matrix form:

$$
\underbrace{\left[\begin{array}{cccc}
I_{2 \kappa+2} & I_{2 \kappa+1} & \cdots & I_{1} \\
I_{2 \kappa+3} & I_{2 \kappa+2} & \cdots & I_{2} \\
\vdots & \vdots & \cdots & \vdots \\
I_{N} & I_{N-1} & \cdots & I_{N-2 \kappa-1}
\end{array}\right]}_{\mathbf{R}} \underbrace{\left[\begin{array}{c}
P_{0} \\
P_{1} \\
\vdots \\
P_{2 \kappa+1}
\end{array}\right]}_{\mathbf{P}}=0,
$$

where frame index $m$ of $I$ in Eq. (1) has been changed to a subscript of $I$ in Eq. (5). Equation (5) shows that at least $N \geq 4 \kappa+2$ samples are required for extraction of the roots of polynomial $P(z)$, enabling us to find the unknown value $z_{k}$. Phase step $\alpha$ at each pixel location can hence be computed from $\alpha=\Re\left(\ln z_{1} / i\right)$.

In many cases $\kappa$ is unknown and can be determined ${ }^{8}$ by observation of the singular-value decomposition (SVD) of the $\mathbf{R}$ matrix in Eq. (5). For a noiseless signal the SVD $\mathbf{R}=\mathbf{U S V}^{T}$ results in a diagonal matrix $\mathbf{S}$ with $2 \kappa+1$ nonzero and $N-2 \kappa-1$ zero singular values, where $\mathbf{U}$ and $\mathbf{V}$ are unitary matrices. If the data are noisy, the $M=2 \kappa+1$ principal values of $\mathbf{S}$ will still tend to be larger than the $N-M$ values, which were originally zero. In addition, the $M$ eigenvectors that correspond to the $M$ eigenvalues of $\mathbf{R}^{T} \mathbf{R}$ are less susceptible to noise pertubations than the remaining $N-M$ eigenvectors. Figure 1 illustrates typical singular values for $\mathbf{S}$ obtained from the SVD of matrix $\mathbf{R}$ for noise at a signal-to-noise ratio (SNR) of $10 \mathrm{~dB}$ and without noise, and when $\kappa=2$ in Eq. (1). Although 18 frequencies were assumed to be present during the estimation, only five principal values of $\mathbf{S}$ for noisy and noiseless signals show a distinctly larger magnitude than the remaining values. The plot thus permits a reliable estimation of the number of harmonics.

There are various sources of noise ${ }^{9}$ in optical interferometry, and the relative contribution of each noise source depends on the system used and on its application. The use of a denoising technique is thus an im- portant step in reducing the effect of noise. In what follows, we consider the case in which white Gaussian noise has been added to the signal. We apply a truncated $\mathrm{SVD}^{10,11}$ to enhance the signal. It involves the following steps: Matrix $\mathbf{R}$ is written in Hankel matrix form, say, $\hat{\mathbf{R}}$, and the SVD of $\hat{\mathbf{R}}=\hat{\mathbf{U}} \overline{\mathbf{S}} \hat{\mathbf{V}}^{T}$ shows the $M$ nonsingular principal values of $\overline{\mathbf{S}}$, which are significantly different from zero. After the nonsignificant $N-M$ singular values of $\overline{\mathbf{S}}$ are set to zero, a matrix $\hat{\mathbf{S}}$ is formed. A denoised matrix $\mathbf{Z}_{M}=\hat{\mathbf{U}} \hat{\mathbf{S}} \hat{\mathbf{V}}^{T}$, which approximates $\hat{\mathbf{R}}$ in the least-squares sense, is then obtained by use of the first $M$ columns of $\hat{\mathbf{U}}, \hat{\mathbf{S}}$, and $\hat{\mathbf{V}}$; $\hat{\mathbf{U}}$ and $\hat{\mathbf{V}}$ are unitary matrices. Finally, a denoised signal $\bar{I}_{m}$ is retrieved by arithmetic averaging along the antidiagonals (or diagonals) of $\mathbf{Z}_{M}$ as follows:

$$
\begin{aligned}
\bar{I}_{m} & =\frac{1}{r-q+1} \sum_{j=1}^{N} \mathbf{Z}_{M} \quad(m-j+1, j), \\
m & =1, \ldots, N,
\end{aligned}
$$

where $r=\max [1, m-$ number of rows $(\hat{\mathbf{R}})+1]$ and $q=\min [$ number of rows $(\hat{\mathbf{R}}), m]$. Denoised signal $\bar{I}_{m}$ is subsequently substituted into Eq. (4).

The concept of high resolution is tested by simulation of the fringe pattern in Eq. (1); we assume that $\kappa=2, \alpha=\pi / 4, a_{ \pm 1, \pm 2}=0.5$, and $\beta_{0}=1$. Additive white Gaussian noise with a SNR of $0-100 \mathrm{~dB}$ is added to Eq. (1) and for each noise-level phase step is computed at every pixel of the data frame without and with application of the denoising described in the previous paragraph. Figures 2(a) and 2(b) show plots for $\alpha$ at any arbitrary pixel location, without and with a denoising procedure, respectively, for 15 data frames. Figure 2(b) shows that denoising is an important step in extracting information from noisy data. The accuracy in the determination of the number of harmonics $\kappa$ and of phase step $\alpha$ in the presence of increasing noise can be improved with an increase in the redundancy of the data frames.

Once phase step $\alpha$ is estimated by the highresolution technique, parameter $\beta_{k}$ can be solved by use of a linear Vandermonde system of equations

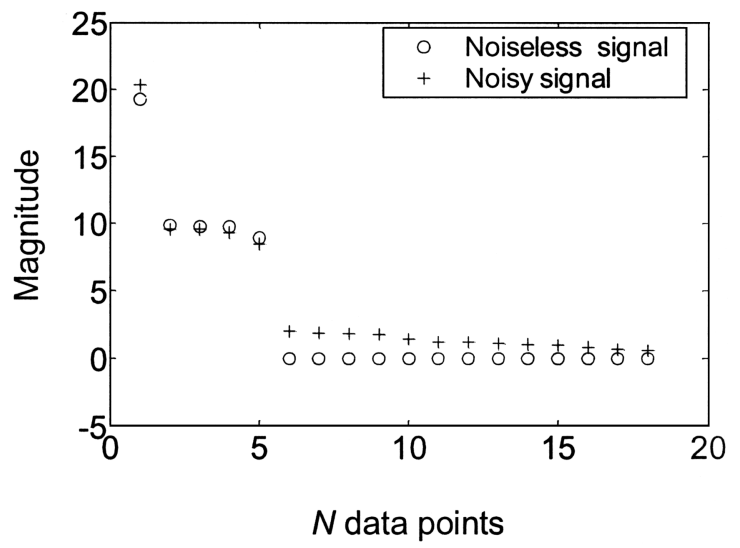

Fig. 1. SVD of $\mathbf{R}$ for a SNR of $20 \mathrm{~dB}$ and without noise. Estimation of the value of $\kappa$ is straightforward for a noiseless signal. 

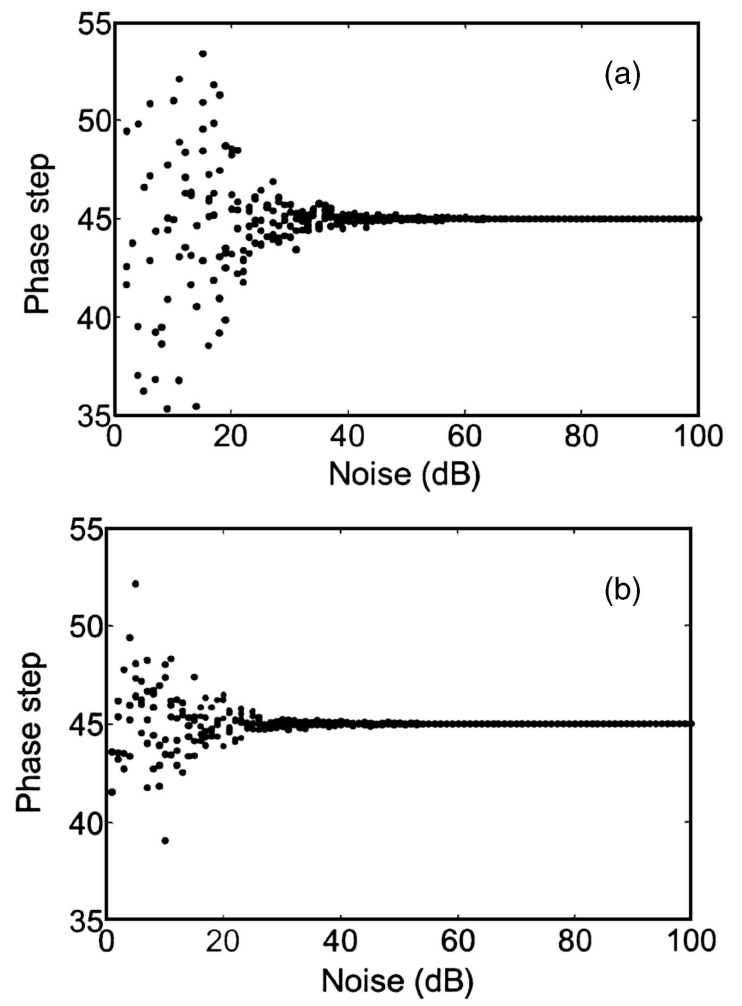

Fig. 2. Phase step $\alpha$ (in degrees) at arbitrary pixel locations versus noise (a) without and (b) with application of the denoising procedure.

obtained from Eq. (2). The matrix thus obtained can be written as
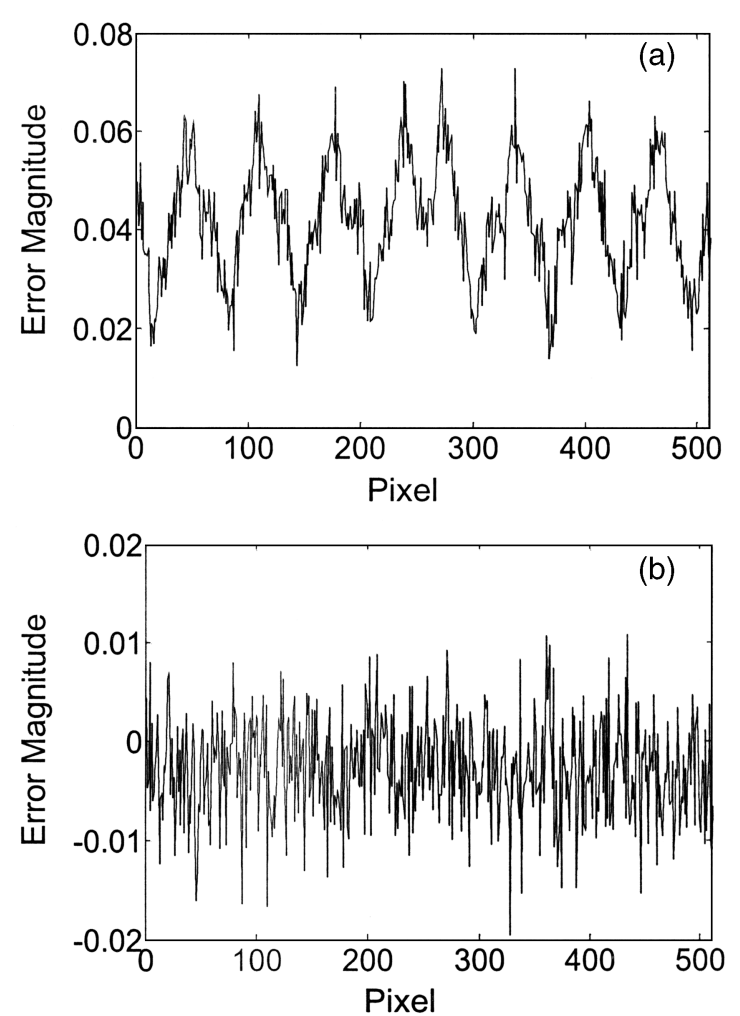

Fig. 3. Error in phase computation (in radians) versus pixel number in the presence of a signal, with a SNR of $30 \mathrm{~dB}$ (a) without and (b) with application of the denoising procedure.

ful discussions on spectral estimation. P. Rastogi's e-mail address is pramod.rastogi@epfl.ch. $\left[\begin{array}{ccccc}\exp \left(i \kappa \alpha_{1}\right) & \exp \left(-i \kappa \alpha_{1}\right) & \exp \left[i(\kappa-1) \alpha_{1}\right] & \cdots & 1 \\ \exp \left(i \kappa \alpha_{2}\right) & \exp \left(-i \kappa \alpha_{2}\right) & \exp \left[i(\kappa-1) \alpha_{2}\right] & \cdots & 1 \\ \vdots & \vdots & \vdots & \cdots & \vdots \\ \exp \left(i \kappa \alpha_{N}\right) & \exp \left(-i \kappa \alpha_{N}\right) & \exp \left[i(\kappa-1) \alpha_{N}\right] & \vdots & 1\end{array}\right]\left[\begin{array}{c}\beta_{\kappa} \\ \beta_{\kappa}{ }^{*} \\ \vdots \\ \beta_{0}\end{array}\right]=\left[\begin{array}{c}I_{1} \\ I_{2} \\ \vdots \\ I_{N}\end{array}\right]$ where $\alpha_{1}, \alpha_{2}, \ldots, \alpha_{N}$ are phase steps in frames 1 , $2, \ldots, N$, respectively. Phase $\varphi$ is subsequently computed from the argument of $\beta_{1}$. Figures 3(a) and 3(b) show typical errors in the computation of wrapped phase maps of $512 \times 512$ pixels, without and with the denoising step, respectively, and assuming a SNR of $30 \mathrm{~dB}$.

In conclusion, we have proposed a new generalized approach to recovering phase distribution in the presence of PZT miscalibration and higher-order harmonics. The method, by facilitating a free choice of phase shifts from 0 to $\pi$, overcomes the limitation exhibited by previously suggested methods. The proposed technique works well with both diverging and converging beams, as it retrieves the phase steps pixelwise before applying them to the Vandermonde system of equations.

This research is funded by the Swiss National Science Foundation. We thank Thierry Blu for fruit-

\section{References}

1. Y. Surrel, Appl. Opt. 32, 3598 (1993).

2. Y. Surrel, Appl. Opt. 35, 51 (1996).

3. P. Carré, Metrologia 2, 13 (1966).

4. K. G. Larkin and B. F. Oreb, J. Opt. Soc. Am. A 9, 1740 (1992).

5. K. Hibino, B. F. Oreb, D. I. Farrant, and K. G. Larkin, J. Opt. Soc. Am. A 12, 761 (1995).

6. P. Stoica and R. Moses, in Introduction to Spectral Analysis (Prentice-Hall, Englewood Cliffs, N.J., 1997), Chap. 4.

7. J. J. Fuchs, IEEE Trans. Acoust. Speech Signal Process. 36, 1846 (1988).

8. R. Kumaresan and D. W. Tufts, IEEE Trans. Aerosp. Electron. Syst. AES-19, 134 (1983).

9. K. B. Hill, S. A. Basinger, R. A. Stack, and D. J. Brady, Appl. Opt. 36, 3948 (1997).

10. M. Dendrinos, S. Bakamidis, and G. Carayannis, Speech Commun. 10, 45 (1991).

11. P. C. Hansen and S. H. Jensen, IEEE Trans. Signal Process. 46, 1737 (1998). 